\title{
Images Are Data: A Breast Imaging Perspective on a Contemporary Paradigm
}

\section{Bilder sind Daten: Die Perspektive der Brustbildgebung auf ein zeitgenössisches Paradigma}

Authors

Matthias Dietzel', Paola Clauser², Panagiotis Kapetas², Rüdiger Schulz-Wendtland ${ }^{1}$, Pascal Andreas Thomas Baltzer ${ }^{2}$

Affiliations

1 Department of Radiology, University Hospital Erlangen, Germany

2 Department of Biomedical Imaging and Image-Guided Therapy, Division of Molecular and Gender Imaging, Medical University Vienna, Vienna, Austria

Key words

breast, mammography, ultrasound, screening, MR imaging, artificial intelligence

received 20.11.2020

accepted 22.12.2020

published online 03.02.2021

Bibliography

Fortschr Röntgenstr 2021; 193: 898-908

DOI 10.1055/a-1346-0095

ISSN 1438-9029

(c) 2021. Thieme. All rights reserved.

Georg Thieme Verlag KG, Rüdigerstraße 14,

70469 Stuttgart, Germany

Correspondence

Prof. Pascal Andreas Thomas Baltzer

Department of Biomedical Imaging and Image-Guided

Therapy, Division of Molecular and Gender Imaging,

Medical University of Vienna, Friedrich-Schiller-University

Jena, Währinger Gürtel 18-20, 1090 Vienna, Austria

Tel.: $+43 / 3641 / 9324801$

Fax: $+43 /(0) 1 / 4040048180$

patbaltzer@gmail.com

\section{ZUSAMMENFASSUNG}

Hintergrund Radiologische Untersuchungen nicht nur als bloße Bilder, sondern als Datenquelle zu betrachten, ist zum modernen Paradigma der diagnostischen Bildgebung geworden. Dieser Perspektivwechsel hat sich besonders in der Brustbildgebung durchgesetzt, ermöglicht er doch aus der Informatik abgeleitete Verfahren anzuwenden, innovative klinische Anwendungen zu realisieren und bereits etablierte Methoden zu verfeinern. In diesem Zusammenhang sind die Begriffe „bildgebender Biomarker“, „Radiomics“ und „künstliche Intelligenz“ von zentraler Bedeutung. Diese Methoden versprechen nichtinvasive, kostengünstige (z. B. im Vergleich zu Multigen-Arrays), Workflow-freundliche (automatisiert, nur eine Untersuchung, sofortige Ergebnisse) und klinisch relevante Informationen.

Methode und Ergebnisse Dieser Artikel wurde als narratives Review zu dem besagten Paradigma im Bereich der Brustbildgebung konzipiert. Der Schwerpunkt liegt auf den Schlüsselkonzepten und wichtigen Schlagworten. Für alle Bereiche der Brustbildgebung werden beispielhafte Studien diskutiert.

Schlussfolgerung Die Interpretation von radiologischen Untersuchungen als Datenquelle verspricht eine Optimierung der Behandlung von Brustkrebspatientinnen im Zeitalter der Präzisionsmedizin, da hiermit die Diagnose verfeinert und eine individualisierte Behandlung erreicht werden könnte.

\section{Kernaussagen:}

- In der konventionellen Brustbildgebung werden Untersuchungen anhand von visuell erkennbaren Mustern interpretiert.

- Das Radiomics-Paradigma behandelt radiologische Brustuntersuchungen hingegen als abstrakte Datenquelle, in der Informationen zu finden sind, die über visuell erkennbare Muster hinausgehen.

- Derartige radiomische Signaturen können als bildgebende Biomarker angesehen werden, da sie diagnostische, prädiktive und prognostische Informationen liefern.

- Derartige bildgebende Biomarker können im Zeitalter der Präzisionsmedizin zur Individualisierung der Brustkrebsbehandlung eingesetzt werden.

- In diesem narrativen Übersichtsartikel stellen wir das Radiomics-Paradigma auf dem Gebiet der Brustkrebsbildgebung anhand von exemplarischen Literaturbeispielen dar.

\section{ABSTRACT}

Background Considering radiological examinations not as mere images, but as a source of data, has become the key paradigm in the diagnostic imaging field. This change of perspective is particularly popular in breast imaging. It allows breast radiologists to apply algorithms derived from computer science, to realize innovative clinical applications, and to refine already established methods. In this context, the terminology "imaging biomarker", "radiomics", and "artificial intelligence" are of pivotal importance. These methods promise 
noninvasive, low-cost (e.g., in comparison to multigene arrays), and workflow-friendly (automated, only one examination, instantaneous results, etc.) delivery of clinically relevant information.

Methods and Results This paper is designed as a narrative review on the previously mentioned paradigm. The focus is on key concepts in breast imaging and important buzzwords are explained. For all areas of breast imaging, exemplary studies and potential clinical use cases are discussed.

Conclusion Considering radiological examination as a source of data may optimize patient management by guiding individualized breast cancer diagnosis and oncologic treatment in the age of precision medicine.

\section{Key Points:}

- In conventional breast imaging, examinations are interpreted based on patterns perceivable by visual inspection.
- The radiomics paradigm treats breast images as a source of data, containing information beyond what is visible to our eyes.

- This results in radiomic signatures that may be considered as imaging biomarkers, as they provide diagnostic, predictive, and prognostic information.

- Radiomics derived imaging biomarkers may be used to individualize breast cancer treatment in the era of precision medicine.

- The concept and key research of radiomics in the field of breast imaging will be discussed in this narrative review.

\section{Citation Format}

- Dietzel M, Clauser P, Kapetas P et al. Images Are Data: A Breast Imaging Perspective on a Contemporary Paradigm. Fortschr Röntgenstr 2021; 193: 898-908

\section{Background}

\section{Breast imaging settings and potential impact of data-driven tools}

Imaging plays a decisive role in the detection, management, and follow-up of breast disease. By definition, breast imaging is multimodal, meaning that a combination of different imaging methods is employed as suited to the clinical workflow. For a better understanding of the results and decisions that may be impacted by data-driven tools, a structured description is helpful.

\section{Screening}

Despite ongoing controversies around screening tests in general and breast cancer screening specifically, most expert societies recommend secondary breast cancer prevention by screening mammography for the early detection of breast cancer [1]. Mass screening applies the same test (mammography) to the eligible population, that is, with some variations, women aged 5070 years. In recent years, medicine has moved to a more personalized approach that recognizes that diagnostic and therapeutic interventions have an individual impact [2]. This also holds true for breast cancer screening: women differ regarding their risk profile and screening mammography is less effective in dense breasts as compared to non-dense breasts as lesions are more likely to be masked by breast parenchyma. In addition, breast density is one independent risk factor that is easily accessible by mammography [3]. A woman with dense breasts is thus at double risk: higher risk of developing cancer compared to the average population and risk of missed cancer by mammography. On the other hand, a subgroup of women may exhibit such a low risk of breast cancer that screening is so unlikely to have an impact on morbidity and mortality that mammography screening may not be required. Thus, one future approach to solve this issue is to use data-driven tools including automated image analyses to stratify women into those who require screening mammography, those who require supplemental or alternative screening tools such as breast MRI, and those who may not require screening at all ( $\triangleright$ Fig. $\mathbf{1})$. The mass screening setting is characterized by a low prevalence of breast cancer and thus an overwhelming proportion of negative (= healthy) cases [4]. Image data-driven tools could help to identify cases that are definitely benign and thus reduce the workload and consequently possible reader mistakes as a result of performing a tiring and monotonous task [5]. The latter could be avoided by computational image-data analysis pointing out evident pathology. A positive finding is a screening recall, usually confirmed or refuted by a second reader and then sent to an assessment center ( $\triangleright$ Fig. 1). Again, data-driven tools can help in this step by acting as a second reader, providing an objective risk of cancer estimation, reducing unnecessary recalls ( $\triangleright$ Fig. $\mathbf{1}$ ).

\section{Assessment}

Both screening recalls in asymptomatic women as well as symptomatic women are assessed by further imaging and, if required, minimally invasive image-guided biopsies. The physician has to decide whether the recall or symptomatic finding requires further imaging or biopsy (and the method of image guidance) or can be downgraded and what follow-up tests and intervals are necessary. Both the choice of further imaging as well as the decision provide ample opportunity to employ data-driven decision-support tools ( $\triangleright$ Fig. 1). From a value-based health care perspective, avoidable biopsies and avoidable additional tests provide value for the patient while at the same time saving resources.

\section{Therapy guidance and monitoring}

Breast cancer treatment is driven by molecular subtypes that are usually determined using immunohistochemically examined tissue specimens from image-guided biopsies. A significant proportion of breast cancer cases undergo neoadjuvant therapy before surgery that can even be omitted or at least delayed in the case of multimorbid patients. As neoadjuvant treatment is a main cost driver in breast cancer diagnosis and treatment [6], imaging tests could play a pivotal role in adapting treatment [7]. While 


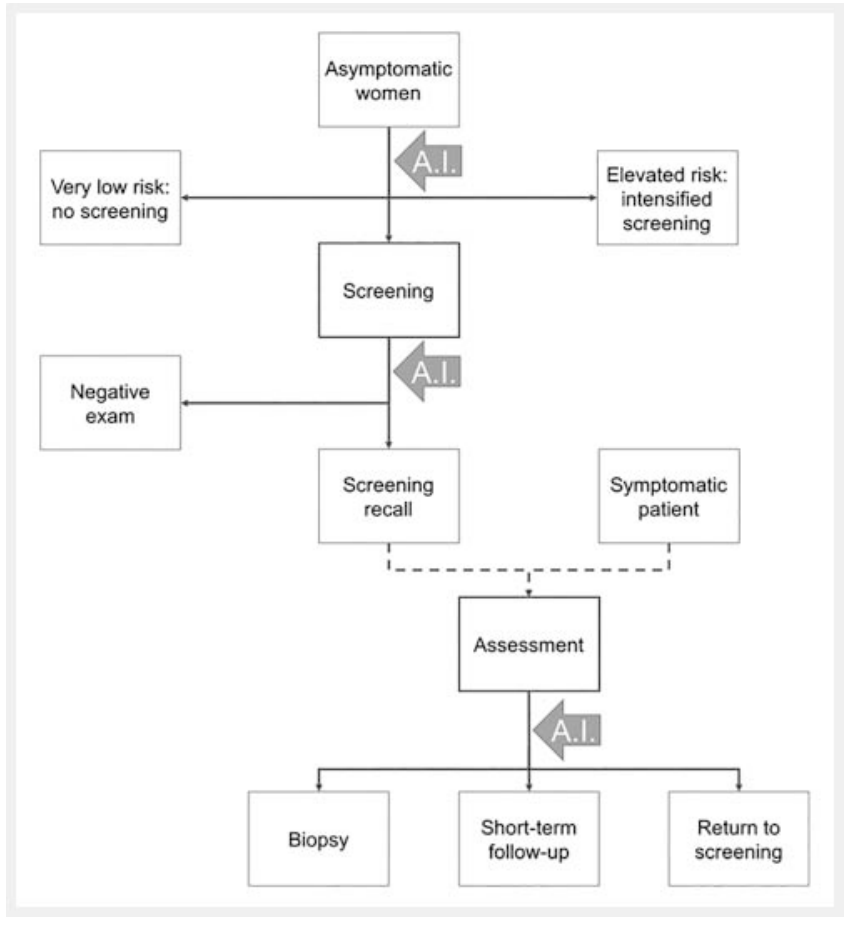

- Fig. 1 Arrows represent decisions, dashed arrows logical steps. Possible interaction steps of data-driven decision support tools are highlighted as grey A.I. arrows.

- Abb. 1 Pfeile stehen für Entscheidungen, gestrichelte Pfeile für logische Schritte. Mögliche Interaktionsschritte von datengesteuerten Werkzeugen zur Entscheidungsfindung sind als graue A.I.-Pfeile hervorgehoben.

therapy could be deescalated in early complete responders, therapy regimes could be changed in non-responders. While there is currently no solid evidence regarding which technology and imaging markers should be used when, it is conceivable that the complexity of the matter would, again, profit from data-driven decision-support tools.

\section{Key buzz words}

Before we address the topic in a narrative overview on the current literature, three important terms are introduced and briefly discussed from the breast imaging perspective.

\section{Breast imaging biomarkers}

The term "biomarker" was initially restricted to biologic molecules [8]. The much broader current definition considers any "defined characteristic that is measured as an indicator of normal biological processes, pathogenic processes, or responses to an exposure or intervention, including therapeutic interventions" as a potential "biomarker" [9]. This conceptual change opened the door to the evaluation of radiologic examinations as an "imaging biomarker" [10].

In clinical practice and breast cancer-related research, biomarkers are used as surrogate outcome measures [11]. Surrogate parameters are of great importance, because direct endpoints (e. g. overall survival) are very difficult to capture in clinical trials [12]. Typical applications of biomarkers are prediction and monitoring of therapy response (e. g., ki67 determination during ongoing neoadjuvant chemotherapy) as well as prognosis of patient outcome (risk stratification using genetic microarrays) [13-17]. Accordingly, biomarkers are considered pivotal in precision medicine [18].

Imaging biomarkers may be classified as quantitative, ordinal, and qualitative. Breast cancer size remains one of the most important surrogates of patient outcome and treatment response, which is why radiological measurement of tumor dimensions is a classic quantitative breast imaging biomarker [19, 20]. Beside morphologic evaluations, breast imaging offers numerous advanced methods of functional tissue assessment. For instance, the tissue microstructure may be quantitatively investigated by the apparent diffusion coefficient (ADC) [21, 22]. Distinguishing invasive and in situ breast cancer growth is pivotal both for therapy planning and for the estimation of patient outcome. According to Bickel et al., the ADC may be used as a quantitative breast imaging biomarker of breast cancer invasiveness [22].

Breast density has been established as one of the most important independent risk factors of breast cancer [3, 23, 24]. In the clinical routine breast density is visually assessed on a four-level scale and thus may be considered an ordinal imaging biomarker [24]. In addition, a variety of methods have been described to assess breast density ranging from fully automated to quantitative techniques [25-28].

Qualitative breast imaging descriptors are typically used to establish the diagnosis of breast cancer [24]. However, many of these patterns could be associated as well with the underlying tumor biology and may be considered qualitative biomarkers. For instance, perifocal edema is predictive of malignancy but also suggestive of unfavorable prognosis such as the presence lymph nodes metastasis [29-31].

\section{Imaging Biomarker Panels}

The discriminatory power of individual breast imaging biomarkers can be further improved if they are combined with one another into imaging biomarker panels. Various methods have been investigated for this purpose, with artificial intelligence and radiomics being most promising [14, 15, 32-34]. For instance, the occurrence of lymph node metastasis may be further improved by the joint analysis of qualitative and quantitative breast imaging biomarkers applying artificial neural networks or radiomics [33, 35-37].

\section{Radiomics}

Among the methods for combined analysis of imaging biomarker panels, radiomics have arguably gained the greatest attention [38]. Since the introduction of the term, to date a total of 3145 articles on radiomics have already been published, of which the majority $(49.4 \%, 1555 / 3145)$ are related to breast imaging [39]. This illustrates both the huge interest among the scientific community in radiomics-related research and the dominant role of breast imaging. 
Radiomics approaches breast images not as mere pictures but as a source of comprehensive data containing viable patient information not perceivable with our human eyes. The analytic radiomic workflow starts with segmentation of the tissue of interest, followed by extraction of a large number of tissue characteristics expressed as arithmetic functions, of which the most important ones are chosen during statistical feature selection and applied for machine-learning-based model building. The output of this workflow is an algorithm or model that can diagnose or predict the intended target condition or property. The result of the radiomic analysis or the specific radiomics signature is typically expressed as a numeric value (the predicted probability of the target condition being met) and may be regarded as a quantitative imaging biomarker [32].

In clinical practice radiomics may be used for virtually any use case such as distinguishing malignant from benign breast lesions, the prediction of patient outcome, tumor biology, and response to neoadjuvant chemotherapy and examples from the literature are highlighted in > Fig. 2, 3 [36, 40-43].

\section{Artificial intelligence}

Next to radiomics, artificial intelligence (Al) is the emerging topic in radiology [44-48]. Both methods aim at processing information otherwise hidden from human interpretation and may be used in combination [44, 49]. Just like radiomics, Al can be used for virtually any clinical use ranging from distinguishing malignant from benign breast lesions to the prediction of direct patient outcome $[44,50,51]$. Yet, unlike radiomics, the definition of $\mathrm{Al}$ is rather vague. This might be due to the fact that the term (non-artificial) "intelligence" already lacks a generally accepted definition [52]. So commonly, $\mathrm{Al}$ is used as an umbrella term, summarizing various disciplines of computer science, all of which aim to mimic certain cognitive functions and can be as simple as basic multivariable classification algorithms [44-47].

In breast imaging the most investigated subdiscipline of supervised Al is machine learning [44]. Without being explicitly programmed, machine learning algorithms aim to detect ("learn") patterns in imaging data and to link these imaging characteristics to tissue characteristics $[44,46]$. A large variety of methods belong to the machine learning family, such as artificial neural networks (ANNs), support vector machines (SVMs), but also classic statistical procedures, such as decision trees and regression analysis [46]. Each method provides inherent advantages and has been successfully applied to breast imaging research [14, 33, 53, 54].

Without any doubt, $\mathrm{Al}$ in breast imaging goes far beyond supervised machine learning. Further pivotal Al methods such as unsupervised learning and deep learning have already shown high promise for the future development of our field [44, 49, 55-57]. The complexity and importance of this topic call for a dedicated publication summarizing the state of the art of $\mathrm{Al}$ in breast imaging $[18,44,51]$.
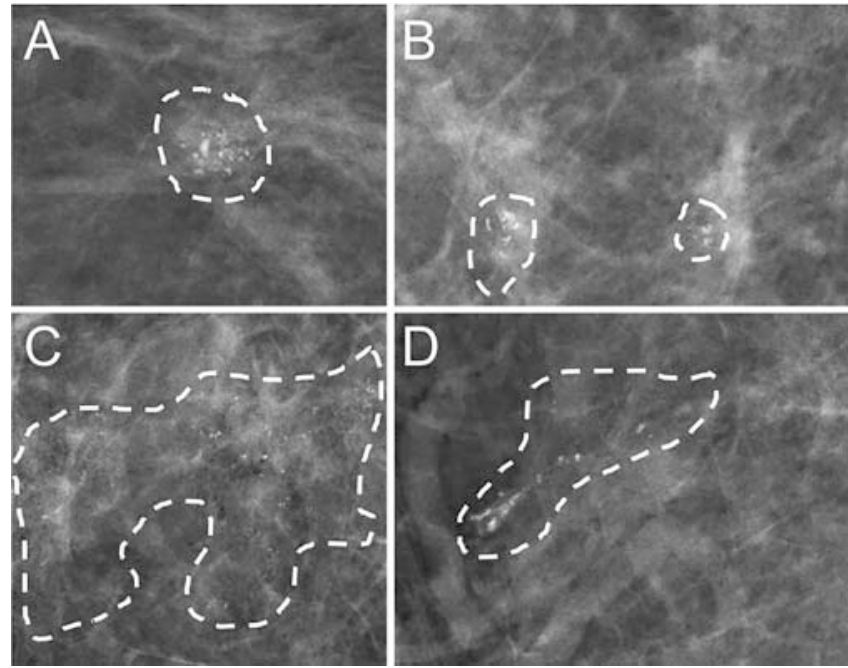

- Fig. 2 Application of radiomics and Al to improve the diagnostic accuracy of breast imaging. Stelzer et al. examined the radiomic signatures of microcalcifications in 235 patients [69]. All findings were assessed as suspicious in conventional visual analysis (BI-RADS IV) and therefore bioptically confirmed. A basic radiomic workflow was applied starting with marking of a region (dashed lines) of interest for data extraction, selection of radiomic features, building and testing the radiomics model. As a result, the authors received a specific radiomic signature, based upon which a biopsy could be safely omitted in up to $45.7 \%$ of the patients. Shown are BI-RADS IV findings correctly classified as benign (A: benign papilloma without atypia; B: fibrocystic changes) or malignant based on the radiomic signature (C: invasive ductal cancer, $\mathbf{D}$ : ductal carcinoma in situ). Results are available in real time, not requiring additional imaging or invasive procedures. Therefore, radiomic signatures may be considered accurate, workflow-friendly, and cost effective which is why they provide value to patient care (modified and reprinted with permission [69]).

- Abb. 2 Stelzer et al. untersuchten die radiomischen Signaturen von Mikroverkalkungen in 235 Patienten [69]. Alle Befunde wurden in der konventionellen visuellen Analyse (BI-RADS IV) als verdächtig bewertet und daher bioptisch bestätigt. Es wurde ein Basis-Workflow angewandt, der mit der Markierung einer für die Datenextraktion interessanten Region (gestrichelte Linien), der Auswahl der radiomischen Merkmale, dem Aufbau und der Prüfung des radiomischen Modells begann. Als Ergebnis erhielten die Autoren eine spezifische radiomische Signatur, auf deren Grundlage bei bis zu 45,7\% der Patienten eine Biopsie sicher vermieden werden konnte. Dargestellt sind BI-RADS-IV-Befunde, die anhand der radiomischen Signatur korrekt als gutartig (A: gutartiges Papillom ohne Atypie; B: fibrozystische Veränderungen) oder bösartig klassifiziert wurden (C: invasiv duktales Karzinom, D: duktales Karzinom in situ). Die Ergebnisse liegen in Echtzeit vor und erfordern keine zusätzliche Bildgebung oder invasive Verfahren. Daher können radiomische Signaturen als genau, Workflow-freundlich und kosteneffektiv angesehen werden, weshalb sie für die Patientenversorgung relevant sind (modifiziert und mit Genehmigung nachgedruckt [69]). 
a

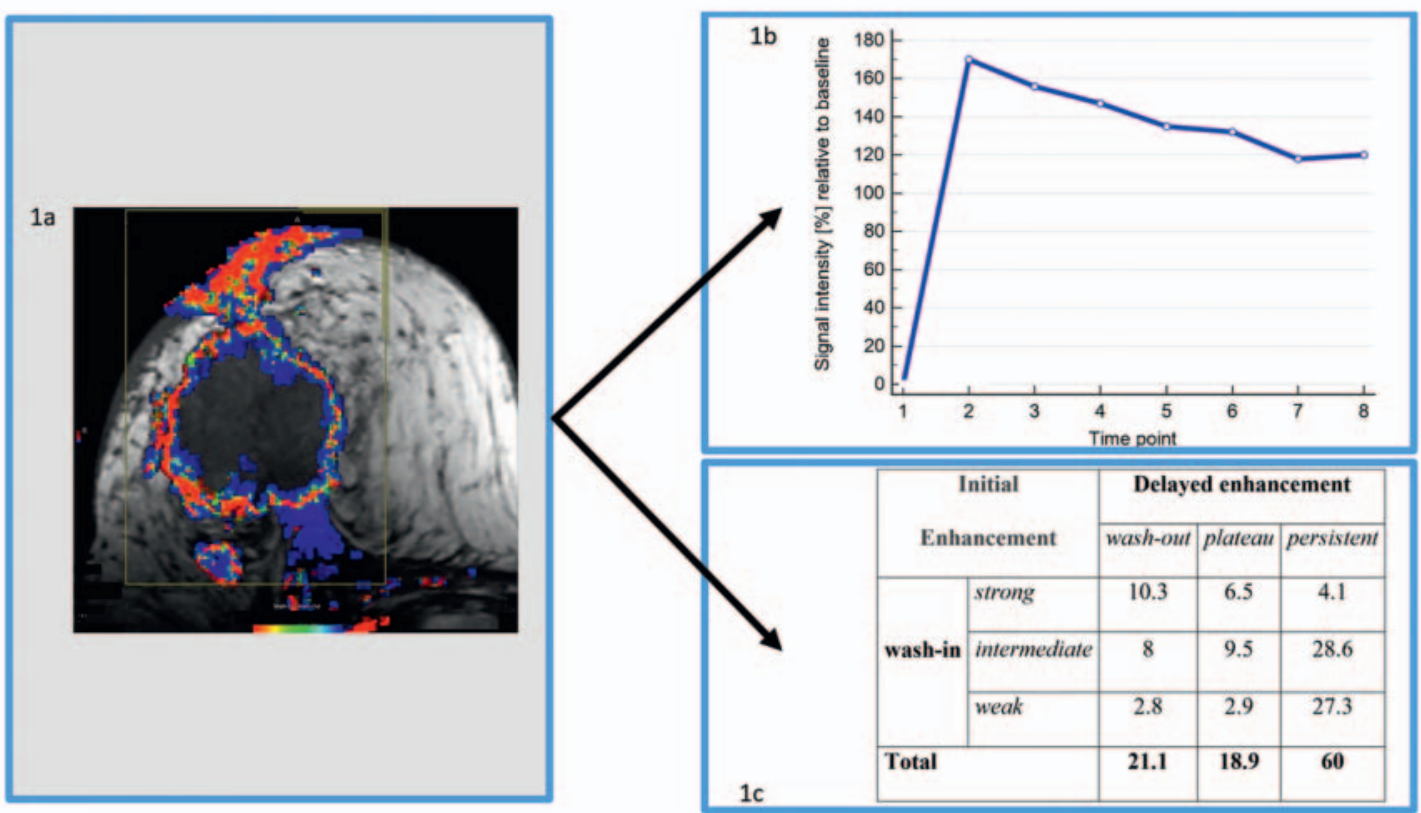

b

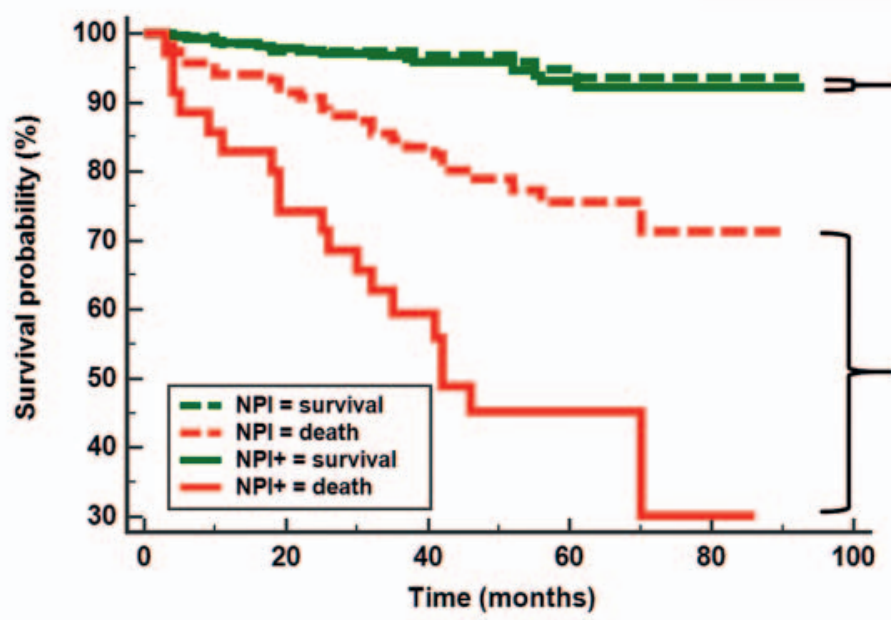

Prediction by $\mathrm{NPI}+$ vs. NPI:

HR= 1.05 (Cl: 0.86-1.27)

$\boldsymbol{P}_{\text {logrank }}=0.65$

\section{DSD}

Prediction by $\mathrm{NPI}+\mathrm{vs}$. NPI:

HR= 4.5 (Cl: $2.14-9.58)$

$\boldsymbol{P}_{\text {logrank }}=0.0001$

- Fig. 3 a Application of radiomics to improve risk stratification of breast cancer patients. Extracts of the radiomics workflow-based breast MRI data are displayed above. Volumetric analysis of dynamic enhancement parameters was performed providing surrogates of tumor heterogeneity and cancer vascularity [17]. Such MRI derived parameters are closely linked to pathophysiology, facilitating interpretation of the radiomic model, a step not always feasible with classic texture parameters. The resulting radiomic signature was integrated into an established prognostic model (Nottingham prognostic index: NPI) yielding NPI+. Upon benchmarking analysis of NPI versus NPI+, the additive benefit of the radiomic signature is evident. Corresponding Kaplan Meier curves illustrate a better risk stratification of NPI+ compared to standard NPI (modified and reprinted with permission [17]). b Application of radiomics to improve risk stratification of breast cancer patients (benchmarking analysis).

- Abb.3 a Oben werden Auszüge aus den auf dem Radiomics Workflow basierenden MRI-Daten der Brust dargestellt. Es wurde eine volumetrische Analyse dynamischer Anreicherungsparameter durchgeführt, die Surrogate der Tumorheterogenität und der Krebsvaskularität liefert [17]. Solche Parameter sind eng mit der Pathophysiologie verbunden und erleichtern die Interpretation des radiomischen Modells; ein Schritt, der mit klassischen Texturparametern nicht immer möglich ist. Die resultierende radiomische Signatur wurde in ein etabliertes Prognosemodell (Nottingham Prognostic Index, NPI) integriert und ergab NPI+. Nach Benchmarking-Analyse von NPI versus NPI+ wird der additive Nutzen der radiomischen Signatur deutlich. Die Kaplan-Meier-Kurven veranschaulichen dort eine bessere Risikostratifizierung von NPI+ im Vergleich zu Standard-NPI (modifiziert und nachgedruckt mit Genehmigung [17]). b Anwendung von Radiomics zur Verbesserung der Risikostratifizierung von Brustkrebspatientinnen (Benchmarking Analyse). 


\section{Current status and future development: A modality-based approach}

\section{Mammography}

The idea of using computer algorithms to process mammography-derived imaging data is not as new as it seems. In fact, this research topic has been investigated since the 1980s [58]. The first studies on computer-aided detection (CAD) systems aimed at supporting the radiologist in the early detection of breast cancer on digital mammography. However, the widespread use of these systems was initially limited by their low specificity and high number of false-positive findings, which reduced the positive effect on the screening readings [59]. The introduction of advanced $\mathrm{Al}$ methods and the availability of large databases together with the framework of international multireader studies reshaped and improved the performance of CAD systems [60].

\section{Lesion characterization}

Recent studies showed that Al-based systems may have a performance not inferior to that of experienced breast radiologists $[60,61]$. Instead of completely replacing the human reader, AI might also be used to switch from double reading to single reading in screening. In this setting the second reader will be substituted by $\mathrm{Al}$ [50]. Another promising clinical use case of $\mathrm{Al}$ is to triage screening mammograms as cancer-free. In this scenario, Al might potentially reduce the radiologist's workload and gain extra time for the read of more cases and/or the assessment of challenging cases [5, 62, 63]. Furthermore, the same algorithms applied for mammography seem to perform equally well for digital breast tomosynthesis (DBT). This finding might be essential for reducing the increased workload related to the use of DBT for instance in screening $[64,65]$.

Irrespective of these encouraging results, a long list of methodological, empirical, ethical, and forensic issues needs to be solved $[66,67]$. Accordingly, we do not expect these systems to completely replace radiologists in the foreseeable future. Moreover, the most realistic use in the diagnostic setting is the application of radiomics and $\mathrm{Al}$ as decision support tools in the assessment for instance in challenging cases, such as the assessment of microcalcifications and BI-RADS IV lesions as demonstrated in - Fig. $2[68,69]$.

\section{Beyond lesion characterization}

Increased breast density is a factor that increases the risk of missing a cancer on mammography, due to tissue superimposition, and also is among the most important risk factor for developing breast cancer [3]. Historically breast density has been evaluated in a semi-quantitative manner using the American College of Radiology Breast Imaging Reporting and Data System [24]. Despite the overall good inter-reader agreement in the assessment of breast density with this semiquantitative method [25], a more accurate and repeatable evaluation of density might improve and standardize the identification of women with dense breast tissue and a subsequently increased risk of breast cancer [26]. Several machine learning-based methods have been developed in the last years, and several studies have shown an association between breast cancer risk and breast density measured using these automated devices [27]. These automated calculations are still subject to variation related to image acquisition (i.e., compression), vendor, and other physical properties [28], but provide a reliable tool for a standardized and repeatable evaluation of breast density $[26,27]$.

Beyond the assessment of breast density, radiomics and AI may also predict lymph node metastasis and HER2neu status [35, 36, $40,42]$. Notably, the additional information derived from the application of an intravenous contrast agent seems to be able to further improve the performance of these algorithms warranting future investigation $[36,40]$.

\section{Ultrasound}

Breast ultrasound (US) is a valuable adjunct to mammography, with a high sensitivity for breast cancer [70]. With the use of advanced techniques, besides B-mode, a US examination can provide information about further tissue properties, such as stiffness (elastography) or vascularity (Doppler, contrast-enhanced US (CEUS)). Radiomics features can be extracted from all these techniques, not only in order to differentiate benign from malignant lesions, but also to identify factors with a prognostic and/or predictive value for breast cancer.

\section{Lesion characterization}

Attempts to use breast US for the extraction of radiomic data begun in the early ' $90 \mathrm{~s}$ with the performance of texture analysis studies and yielded good results for the distinction of benign from malignant breast lesions. Already in 1993, Garra et al. achieved a sensitivity of $100 \%$ and a specificity of $73 \%$ by using textural features derived from co-occurrence matrices [71]. Initial attempts aimed at the development of CAD systems, which at that time demonstrated an increase in sensitivity at the cost of a decreased specificity, especially for experienced readers [72].

Advances in artificial intelligence methods during the last decade allowed for more sophisticated use of US-based radiomic data. Marcon et al. applied a machine learning algorithm to textural features acquired from automated breast ultrasound and achieved an accuracy of $90.7 \%$ for the differentiation of benign from malignant lesions [73]. Lee et al. developed a radiomics score for the discrimination of fibroadenomas from triple-negative carcinomas, which demonstrated an AUC of up to 0.853 , noting however that the performance of the score was dependent on the type of US machine used [41].

Several attempts have been made to apply radiomics to advanced US techniques in order to improve lesion characterization. Zhang Q. et al. used radiomics features derived from strain elastography examinations and coined the term "sonoelastomics" [74]. In their study, seven such features achieved an AUC of 0.917 for the identification of breast malignancies. A more recent study by Zhang $X$. et al. compared deep-learning based radiomics scores acquired from B-mode US and shear-wave elastography (SWE) with the results of the BI-RADS assessment and quantitative SWE parameters and found a significant increase in the diagnostic performance by using radiomics scores, reaching an AUC of 1 for both 
[75]. On the preclinical level, Theek et al. performed a radiomics analysis of CEUS data from different mouse tumor models, achieving correct classifications in $82.1 \%$ of cases [76].

Irrespective of these encouraging results and similar to mammography, ultrasound-derived radiomics is unlikely to replace the human reader in the near future and the biggest clinical advantage is to be expected with respect to decision support [77].

\section{Beyond lesion characterization}

Several attempts have been made to identify breast cancer-related imaging biomarkers based on US-derived radiomics features. Guo et al. reported that different molecular subtypes of breast cancer show differences in several radiomics features [78]. In their study, triple-negative carcinomas could be distinguished from other subtypes with an AUC of 0.760 , based on a feature combination. On the other hand, a series of studies have focused on the prediction of axillary lymph node metastases in breast cancer patients $[49,79]$. Yu et al. developed a nomogram combining US radiomics and clinical features, which could accurately predict the lymph node status with an AUC of 0.81 [80], while Zheng et al. was able to accurately distinguish between a high and a low axillary burden using a combination of clinicopathologic data and radiomics features derived from B-mode US and SWE [55].

\section{Challenges and perspectives of US-based radiomics}

Although US is routinely implemented in breast diagnostics, its use for radiomics studies clearly lags behind that of other modalities, such as MRI or mammography [38]. One of the main reasons is the inherent high inter-examiner variability of US images. US image quality depends on both the acquisition process as well as the examiner's experience while there is also substantial variability between different US devices, as has also been demonstrated by different studies. Moreover, the quality of US images is usually further reduced due to the presence of noise and artifacts. Finally, there is a paucity of large publicly available, labeled, anonymized datasets that would be mandatory for radiomics studies. Keeping these limitations in mind, breast US with its ubiquitous presence, its practicality, and the possibility to acquire a multitude of different morphological and functional information at a low cost has significant potential for application in the definition of breast tumors radiomic signatures and may thus play a substantial role in precision medicine.

\section{PET}

Positron emission tomography (PET)-CT with fluorodeoxyglucose (18F-FDG) is mostly indicated for inconclusive CT findings and high-risk patients and plays only an ancillary role in the staging of breast cancer [81]. Yet, after the introduction of PET-MRI, the interest in PET for local and distant breast cancer staging has increased.

\section{Local Staging}

The use of 18F-FDG PET-MRI has been proposed for local staging in women with breast cancer, but the additional information derived from the PET examination was only marginal in the first studies $[82,83]$. Further analysis showed that MRI and PETderived data could yield relevant additional information. In particular, several studies applied radiomics to the PET-MRI images of the breast and proved that Al could be used to characterize breast lesions and define tumor aggressiveness [84]. Similar to breast density in mammography, background parenchymal enhancement and background parenchymal uptake may be regarded as potential imaging biomarkers for breast cancer incidence [85]. Texture analysis and radiomics could also play a role in the evaluation of tumor aggressiveness as well in the prediction of response to neoadjuvant chemotherapy [86-88]. However, only few single center studies are available on PET MRI-derived imaging biomarkers for local breast cancer staging. More research in this field is needed.

\section{Distant Staging}

PET-MRI is currently rarely used for whole-body staging of newly diagnosed breast cancer. Increasing evidence suggests that the examination could improve sensitivity, as compared to PET-CT, but the limited availability and the high costs of the examination suggest that careful patient selection is warranted [89]. Currently, no large studies have been performed using Al to detect or characterize distant metastasis in patients with breast cancer, but animal studies suggest that the combined information from MRI, PET and Al could improve the early detection of metastasis [90].

\section{MRI}

\section{Lesion characterization}

In 2003, Gibbs et al. reported the potential of quantitative multivariate texture analysis to distinguish benign from malignant lesions in breast MRI [91]. This paper anticipated the key concepts of lesion characterization by breast MRI radiomics by many years and key results have been validated ever since [32, 54, 92-95].

For instance, machine learning was used to generate the Kaiser score. Based on qualitative BI-RADS MRI descriptors, the Kaiser score is a typical ordinal imaging biomarker. It allows estimation of the individual likelihood of breast cancer and specific underlying tumor biology $[54,96]$. Results have been validated by multiple centers in different clinical scenarios, underlining the potential of Al-derived decision support in clinical patient management (for instance [94]).

Whereas the Kaiser score is based on visually extracted qualitative features, numerous authors investigated quantitative features for breast MRI lesion characterization [92, 93, 95]. Yielding similar results compared to the Kaiser score, Parekh and Jacobs developed a radiomic framework using advanced machine learning and multiparametric MRI. In a cohort of 124 patients, they were able to semiautomatically distinguish benign from cancerous lesions achieving an AUC $\leq 0.91$ [95]. As small lesions in breast MRI can be difficult to characterize by visual analysis [97], Gibbs et al. investigated radiomics signatures to characterize subcentimeter breast lesions. Applying support vector machine analysis, authors reported AUC levels ranging from 0.75-0.81 [93].

Benchmarking analysis refers to the comparison of radiomics and $\mathrm{Al}$ results with an established gold standard (see $>$ Fig. $\mathbf{3}$ ) 
[98-100]. Unlike many researchers (e. g. [93, 95]), Bickelhaupt et al. included a benchmarking analysis in a pivotal paper on radiomics signatures derived from diffusion MRI. The authors report promising performance of the radiomics models ( $A U C \leq 0.85$ ), which were, however, clearly outperformed by human readers (AUC $=0.96)$ [92]. Such benchmarking analysis enables us to estimate the practical value of radiomics and $\mathrm{Al}$ in the clinic by providing context. Consequently, benchmarking is considered mandatory for clinical validation of novel imaging biomarkers [98-100].

\section{Beyond lesion characterization}

Breast MRI seems ideally suited for imaging biomarker research, as it provides comprehensive multiparametric data on pathophysiological tumor characteristics [15, 34, 101, 102]. Consequently, a large number of papers are available, demonstrating the predictive and prognostic potential of radiomics and/or Al-derived MRI analysis $[9,101]$.

Prediction of treatment response is pivotal in the management of breast cancer and radiomics. Granzier et al. summarized the literature on breast cancer response prediction to neoadjuvant systemic therapy using MRI-based radiomics. The authors report a high diagnostic accuracy for this diagnostic task (AUC $\leq 0.94$ ) [15]. The prediction of therapy response also depends on the receptor status of the breast cancer [103]. In this respect, the results of Wu et al. are remarkable. They were able to predict the molecular subtype based on BI-RADS features in a multi-modal radiomics analysis ( $\mathrm{f} 1$-score $<87.9 \%$ ) [101]. Based on steroid receptor status, HER2neu status, and ki67 analysis, the molecular subtype has significant prognostic and predictive power and significantly influences the choice of therapy for breast cancer, which is why the results of Wu et al. are clinically relevant $[101,103]$.

One of the most important prognostic factors for patient outcome is lymph node status [19]. Based on visually extracted MRI descriptors, artificial intelligence may be able to identify MRI patterns suggestive of lymph node metastases [33, 37]. Continuing this early research, Liu et al. investigated quantitative radiomics for the prediction of sentinel lymph node metastasis. The diagnostic accuracy exceeded the level achievable with qualitative features (AUC $=0.74$ vs. 0.81) $[33,102]$. Notably Liu et al. could further improve the radiomics model by integrating clinicopathological biomarkers (AUC $=0.81$ vs. 0.87) [102].

Multigene assays represent advanced prognostic and predictive biomarkers of breast cancer. A disadvantage of this approach are the high costs, which is why Bhargava et al. propose alternative methods to safely forgo multigene assays [104]. According to the results of Li et al., MRI radiomics correlate to a certain extent with commercially available multigene assays $(r=0.5-0.56)$ [105]. In analogy to the approach of Bhargava et al., MRI radiomics could also be used to select patients that would benefit from an advanced multi-gene assay [104, 105].

Already in 2003, Boné et al. reported that the combined analysis of qualitative enhancement pattern can predict disease-free survival of breast cancer [106]. Results have since been reproduced by many authors such as Kim et al., Pickles et al., and Baltzer et al. using various radiomics methods and study endpoints (diseasefree, recurrence-free survival, and overall survival) [17, 43, 107, 108]. Some authors benchmarked MRI-derived imaging biomarkers with established prognosis tools $[17,106]$. By benchmarking MRI-derived radiomics with the Nottingham prognostic index (NPI), we could demonstrate that our prognostic radiomics model performed equally well in predicting overall survival of breast cancer patients. More important, the combined analysis of MRI radiomics and the NPI improved the predictive accuracy. These findings underline the potential of creating synergistic effects, if biomarkers are used in combination [17].

\section{Discussion and conclusions}

Considering radiological examinations not as mere images but as a source of data has become the key paradigm in the diagnostic imaging field. This change of perspective allows radiologists to apply methods derived from computer science, to realize innovative clinical applications, and to refine already established methods. In this context, the terms "imaging biomarker", "radiomics", and "artificial intelligence" are of pivotal importance. These methods promise noninvasive, low-cost (e.g. in comparison to multigene arrays) and workflow-friendly (automated, only one examination, instantaneous results) delivery of clinically relevant information [38]. For all areas of breast imaging, exemplary studies were highlighted reporting a variety of clinical use cases. Ultimately, these data may provide value by optimizing patient management by guiding individualized breast cancer diagnosis and treatment in the age of precision medicine.

Yet, a closer look into the literature shows that there are expectations regarding promising technologies that are unlikely to be fulfilled in the foreseeable future $[109,110]$. In order to close the remaining research gaps, considerable efforts from the scientific community are necessary. However, the successful translation of this new imaging paradigm into clinical action requires much more than empirical evidence.

Many stakeholders are making claims on the field such as mass media, patients, and colleagues from non-radiological disciplines and the industry. Taking the lead in this innovation process goes far beyond the role of individual research institutions. It requires communication strategies, joint research projects between academia and vendors, and educational formats accessible to large radiological societies. We are convinced that only in this way can radiomics and $\mathrm{Al}$ be translated into the clinical routine and develop their promising advantage in order to improve patient care. In breast cancer diagnosis and treatment, we have highlighted typical workflow steps that would benefit from these new tools, providing value by improved selection of diagnostic tools and therapeutic strategies.

\section{Conflict of Interest}

The authors declare that they have no conflict of interest. 


\section{References}

[1] Sardanelli F, Aase HS, Álvarez M et al. Position paper on screening for breast cancer by the European Society of Breast Imaging (EUSOBI) and 30 national breast radiology bodies from Austria, Belgium, Bosnia and Herzegovina, Bulgaria, Croatia, Czech Republic, Denmark, Estonia, Finland, France, Germany, Greece, Hungary, Iceland, Ireland, Italy, Israel, Lithuania, Moldova, The Netherlands, Norway, Poland, Portugal, Romania, Serbia, Slovakia, Spain, Sweden, Switzerland and Turkey. Eur Radiol 2017; 27: 2737-2743

[2] Trimboli RM, Giorgi Rossi P, Battisti NML et al. Do we still need breast cancer screening in the era of targeted therapies and precision medicine? Insights Imaging 2020; 11: 105

[3] McCormack VA, dos Santos Silva I. Breast density and parenchymal patterns as markers of breast cancer risk: a meta-analysis. Cancer Epidemiol Biomark Prev Publ Am Assoc Cancer Res Cosponsored Am Soc Prev Oncol 2006; 15: 1159-1169

[4] Baltzer PA, Schelhorn J, Dietzel M et al. Breast screening programs using MRI: is there a role for computer-aided diagnosis? Imaging Med 2010; 2: 659-673

[5] Rodriguez-Ruiz A, Lång K, Gubern-Merida A et al. Can we reduce the workload of mammographic screening by automatic identification of normal exams with artificial intelligence? A feasibility study. Eur Radiol 2019; 29: 4825-4832

[6] Sagar B, Lin YS, Castel LD. Cost drivers for breast, lung, and colorectal cancer care in a commercially insured population over a 6-month episode: an economic analysis from a health plan perspective. J Med Econ 2017; 20: 1018-1023

[7] Tasoulis MK, Lee HB, Yang W et al. Accuracy of Post-Neoadjuvant Chemotherapy Image-Guided Breast Biopsy to Predict Residual Cancer. JAMA Surg 2020; 155: e204103

[8] World Health Organization. Biomarkers In Risk Assessment: Validity And Validation. 2020

[9] FDA-NIH Biomarker Working Group. BEST (Biomarkers, EndpointS, and other Tools) Resource, Silver Spring (MD): Food and Drug Administration (US). 2016

[10] O'Connor JPB, Aboagye EO, Adams JE et al. Imaging biomarker roadmap for cancer studies. Nat Rev Clin Oncol 2017; 14: 169-186

[11] Weaver O, Leung JWT. Biomarkers and Imaging of Breast Cancer. Am J Roentgenol 2017; 210: 271-278

[12] Fleming TR, Powers JH. Biomarkers and Surrogate Endpoints In Clinical Trials. Stat Med 2012; 31: 2973-2984

[13] Chen JH, Baek HM, Nalcioglu O et al. Estrogen receptor and breast MR imaging features: a correlation study. J Magn Reson Imaging JMRI 2008; 27: $825-833$

[14] Dietzel M, Kaiser C, Pinker K et al. Automated Semi-Quantitative Analysis of Breast MRI: Potential Imaging Biomarker for the Prediction of Tissue Response to Neoadjuvant Chemotherapy. Breast Care Basel Switz 2017; 12: $231-236$

[15] Granzier RWY, van Nijnatten TJA, Woodruff HC et al. Exploring breast cancer response prediction to neoadjuvant systemic therapy using MRIbased radiomics: A systematic review. Eur J Radiol 2019; 121: 108736

[16] Ha R, Chang P, Mutasa S et al. Convolutional Neural Network Using a Breast MRI Tumor Dataset Can Predict Oncotype Dx Recurrence Score. J Magn Reson Imaging 2019; 49: 518-524

[17] Dietzel M, Schulz-Wendtland R, Ellmann S et al. Automated volumetric radiomic analysis of breast cancer vascularization improves survival prediction in primary breast cancer. Sci Rep 2020; 10: 1-11

[18] Pinker K, Chin J, Melsaether AN et al. Precision Medicine and Radiogenomics in Breast Cancer: New Approaches toward Diagnosis and Treatment. Radiology 2018; 287: 732-747
[19] Carter CL, Allen C, Henson DE. Relation of tumor size, lymph node status, and survival in 24740 breast cancer cases. Cancer 1989; 63: 181 187

[20] Eisenhauer EA, Therasse P, Bogaerts J et al. New response evaluation criteria in solid tumours: Revised RECIST guideline (version 1.1). Eur J Cancer 2009; 45: 228-247

[21] Baltzer P, Mann RM, lima $M$ et al. Diffusion-weighted imaging of the breast-a consensus and mission statement from the EUSOBI International Breast Diffusion-Weighted Imaging working group. Eur Radiol 2020; 30: 1436-1450

[22] Bickel H, Pinker-Domenig K, Bogner W et al. Quantitative apparent diffusion coefficient as a noninvasive imaging biomarker for the differentiation of invasive breast cancer and ductal carcinoma in situ. Invest Radiol 2015; 50: 95-100

[23] Pettersson A, Graff RE, Ursin G et al. Mammographic density phenotypes and risk of breast cancer: a meta-analysis. J Natl Cancer Inst 2014; 106

[24] D’Orsi C, Sickles E, Mendelson E et al. ACR BI-RADS ${ }^{\circledR}$ Atlas, Breast Imaging Reporting and Data System. American College of Radiology. 2013

[25] Le Boulc'h M, Bekhouche A, Kermarrec E et al. Comparison of breast density assessment between human eye and automated software on digital and synthetic mammography: Impact on breast cancer risk. Diagn Interv Imaging 2020; 101: 811-819

[26] Destounis SV, Santacroce A, Arieno A. Update on Breast Density, Risk Estimation, and Supplemental Screening. Am J Roentgenol 2020; 214: 296-305

[27] Wengert G], Helbich TH, Kapetas P et al. Density and tailored breast cancer screening: practice and prediction - an overview. Acta Radiol Open 2018; 7: 2058460118791212

[28] Gastounioti A, Oustimov A, Keller BM et al. Breast parenchymal patterns in processed versus raw digital mammograms: A large population study toward assessing differences in quantitative measures across image representations. Med Phys 2016; 43: 5862

[29] Baltzer PAT, Yang F, Dietzel $M$ et al. Sensitivity and specificity of unilateral edema on T2W-TSE sequences in MR-Mammography considering 974 histologically verified lesions. Breast J 2010; 16: 233-239

[30] Kaiser CG, Herold M, Krammer ] et al. Prognostic Value of "Prepectoral Edema" in MR-mammography. Anticancer Res 2017; 37: 1989-1995

[31] Cheon H, Kim H], Kim TH et al. Invasive Breast Cancer: Prognostic Value of Peritumoral Edema Identified at Preoperative MR Imaging. Radiology 2018; 287: 68-75

[32] Lambin P, Rios-Velazquez E, Leijenaar R et al. Radiomics: Extracting more information from medical images using advanced feature analysis. Eur J Cancer 2012; 48: 441-446

[33] Dietzel M, Baltzer PAT, Dietzel A et al. Application of artificial neural networks for the prediction of lymph node metastases to the ipsilateral axilla - initial experience in 194 patients using magnetic resonance mammography. Acta Radiol Stockh Swed 1987 2010; 51: 851-858

[34] Dietzel M, Zoubi R, Vag T et al. Association between survival in patients with primary invasive breast cancer and computer aided MRI. J Magn Reson Imaging JMRI 2013; 37: 146-155

[35] Tan H, Wu Y, Bao F et al. Mammography-based radiomics nomogram: a potential biomarker to predict axillary lymph node metastasis in breast cancer. Br J Radiol 2020; 93: 20191019

[36] Mao N, Yin P, Li Q et al. Radiomics nomogram of contrast-enhanced spectral mammography for prediction of axillary lymph node metastasis in breast cancer: a multicenter study. Eur Radiol 2020; 30: 6732-6739

[37] Dietzel M, Baltzer PAT, Vag T et al. Application of breast MRI for prediction of lymph node metastases - systematic approach using 17 individual descriptors and a dedicated decision tree. Acta Radiol Stockh Swed 1987 2010; 51: 885-894 
[38] Song J, Yin $\mathrm{Y}$, Wang $\mathrm{H}$ et al. A review of original articles published in the emerging field of radiomics. Eur J Radiol 2020; 127: 108991

[39] PubMed search: "radiomics" and "breast". 2020

[40] Marino MA, Leithner D, Sung J et al. Radiomics for Tumor Characterization in Breast Cancer Patients: A Feasibility Study Comparing ContrastEnhanced Mammography and Magnetic Resonance Imaging. Diagnostics (Basel) 2020; 10: 492

[41] Lee SE, Han K, Kwak JY et al. Radiomics of US texture features in differential diagnosis between triple-negative breast cancer and fibroadenoma. Sci Rep 2018; 8: 13546

[42] Zhou J, Tan H, Bai Y et al. Evaluating the HER-2 status of breast cancer using mammography radiomics features. Eur J Radiol 2019; 121: 108718

[43] Pickles MD, Lowry M, Gibbs P. Pretreatment Prognostic Value of Dynamic Contrast-Enhanced Magnetic Resonance Imaging Vascular, Texture, Shape, and Size Parameters Compared With Traditional Survival Indicators Obtained From Locally Advanced Breast Cancer Patients. Invest Radiol 2016; 51: 177-185

[44] Codari M, Schiaffino S, Sardanelli F et al. Artificial Intelligence for Breast MRI in 2008-2018: A Systematic Mapping Review. Am J Roentgenol 2019; 212: 280-292

[45] Fazal MI, Patel ME, Tye J et al. The past, present and future role of artificial intelligence in imaging. Eur J Radiol 2018; 105: 246-250

[46] van Assen M, Lee SJ, De Cecco CN. Artificial intelligence from A to Z: From neural network to legal framework. Eur J Radiol 2020; 129: 109083

[47] van Hoek J, Huber A, Leichtle A et al. A survey on the future of radiology among radiologists, medical students and surgeons: Students and surgeons tend to be more skeptical about artificial intelligence and radiologists may fear that other disciplines take over. Eur J Radiol 2019; 121: 108742

[48] Liew C. The future of radiology augmented with Artificial Intelligence: A strategy for success. Eur J Radiol 2018; 102: 152-156

[49] Sun Q, Lin X, Zhao Y et al. Deep Learning vs. Radiomics for Predicting Axillary Lymph Node Metastasis of Breast Cancer Using Ultrasound Images: Don't Forget the Peritumoral Region. Front Oncol 2020; 10: 53

[50] Schaffter T, Buist DSM, Lee Cl et al. Evaluation of Combined Artificial Intelligence and Radiologist Assessment to Interpret Screening Mammograms. JAMA Netw Open 2020; 3: e200265

[51] Sheth D, Giger ML. Artificial intelligence in the interpretation of breast cancer on MRI. J Magn Reson Imaging 2020; 51: 1310-1324

[52] Legg S, Hutter M. A Collection of Definitions of Intelligence. In: Proc 2007 Conf. Adv. Artif. Gen. Intell. Concepts Archit. Algorithms Proc. AGI Workshop 2006 1. Aufl. NLD: IOS Press; 2007: 17-24

[53] Ellmann S, Wenkel E, Dietzel M et al. Implementation of machine learning into clinical breast MRI: Potential for objective and accurate decisionmaking in suspicious breast masses. PLOS ONE 2020; 15: e0228446

[54] Baltzer PAT, Dietzel M, Kaiser WA. A simple and robust classification tree for differentiation between benign and malignant lesions in MR-mammography. Eur Radiol 2013; 23: 2051-2060

[55] Zheng X, Yao Z, Huang Y et al. Deep learning radiomics can predict axillary lymph node status in early-stage breast cancer. Nat Commun 2020; 11: 1236

[56] Kumar SN, Fred AL, Varghese PS. Suspicious Lesion Segmentation on Brain, Mammograms and Breast MR Images Using New Optimized Spatial Feature Based Super-Pixel Fuzzy C-Means Clustering. J Digit Imaging 2019; 32: 322-335

[57] Saba L, Biswas M, Kuppili V et al. The present and future of deep learning in radiology. Eur J Radiol 2019; 114: 14-24

[58] Chan HP, Doi K, Galhotra S et al. Image feature analysis and computeraided diagnosis in digital radiography. I. Automated detection of microcalcifications in mammography. Med Phys 1987; 14: 538-548
[59] Lehman CD, Wellman RD, Buist DSM et al. Diagnostic Accuracy of Digital Screening Mammography with and without Computer-aided Detection. JAMA Intern Med 2015; 175: 1828-1837

[60] Rodriguez-Ruiz A, Lång K, Gubern-Merida A et al. Stand-Alone Artificial Intelligence for Breast Cancer Detection in Mammography: Comparison With 101 Radiologists. JNCI J Natl Cancer Inst 2019; 111: 916-922

[61] McKinney SM, Sieniek M, Godbole V et al. International evaluation of an Al system for breast cancer screening. Nature 2020; 577: 89-94

[62] Lång K, Dustler M, Dahlblom V et al. Identifying normal mammograms in a large screening population using artificial intelligence. Eur Radiol 2020. Online ahead of print

[63] Yala A, Schuster T, Miles R et al. A Deep Learning Model to Triage Screening Mammograms: A Simulation Study. Radiology 2019; 293: $38-46$

[64] Geras KJ, Mann RM, Moy L. Artificial Intelligence for Mammography and Digital Breast Tomosynthesis: Current Concepts and Future Perspectives. Radiology 2019; 293: 246-259

[65] Sechopoulos I, Teuwen J, Mann R. Artificial intelligence for breast cancer detection in mammography and digital breast tomosynthesis: State of the art. Semin Cancer Biol 2020: S1044-579X(20)30135-8

[66] Safdar NM, Banja JD, Meltzer CC. Ethical considerations in artificial intelligence. Eur ] Radiol 2020; 122: 108768

[67] Langlotz CP, Allen B, Erickson B] et al. A Roadmap for Foundational Research on Artificial Intelligence in Medical Imaging: From the 2018 NIH/RSNA/ACR/The Academy Workshop. Radiology 2019; 291: 781791

[68] Lei C, Wei W, Liu Z et al. Mammography-based radiomic analysis for predicting benign BI-RADS category 4 calcifications. Eur J Radiol 2019; 121: 108711

[69] Stelzer PD, Steding O, Raudner MW et al. Combined texture analysis and machine learning in suspicious calcifications detected by mammography: Potential to avoid unnecessary stereotactical biopsies. Eur J Radiol 2020; 132: 109309

[70] Berg WA, Zhang Z, Lehrer D et al. Detection of Breast Cancer with Addition of Annual Screening Ultrasound or a Single Screening MRI to Mammography in Women with Elevated Breast Cancer Risk. JAMA J Am Med Assoc 2012; 307: 1394-1404

[71] Garra BS, Krasner BH, Horii SC et al. Improving the Distinction between Benign and Malignant Breast Lesions: The Value of Sonographic Texture Analysis: Ultrason. Imaging 1993; 15: 267-285

[72] Chabi ML, Borget I, Ardiles R et al. Evaluation of the accuracy of a computer-aided diagnosis (CAD) system in breast ultrasound according to the radiologist's experience. Acad Radiol 2012; 19: 311-319

[73] Marcon M, Ciritsis A, Rossi C et al. Diagnostic performance of machine learning applied to texture analysis-derived features for breast lesion characterisation at automated breast ultrasound: a pilot study. Eur Radiol Exp 2019; 3: 44

[74] Zhang Q, Xiao Y, Suo J et al. Sonoelastomics for Breast Tumor Classification: A Radiomics Approach with Clustering-Based Feature Selection on Sonoelastography. Ultrasound Med Biol 2017; 43: 1058-1069

[75] Zhang X, Liang M, Yang Z et al. Deep Learning-Based Radiomics of B-Mode Ultrasonography and Shear-Wave Elastography: Improved Performance in Breast Mass Classification. Front Oncol 2020; 10: 1621

[76] Theek B, Opacic T, Magnuska Z et al. Radiomic analysis of contrast-enhanced ultrasound data. Sci Rep 2018; 8: 11359

[77] de Fleury EFC, Marcomini K. Impact of radiomics on the breast ultrasound radiologist's clinical practice: From lumpologist to data wrangler. Eur J Radiol 2020; 131: 109197

[78] Guo Y, Hu Y, Qiao M et al. Radiomics Analysis on Ultrasound for Prediction of Biologic Behavior in Breast Invasive Ductal Carcinoma. Clin Breast Cancer 2018; 18: e335-e344 
[79] Qiu X, Jiang Y, Zhao Q et al. Could Ultrasound-Based Radiomics Noninvasively Predict Axillary Lymph Node Metastasis in Breast Cancer? J Ultrasound Med 2020; 39: 1897-1905

[80] Yu FH, Wang JX, Ye XH et al. Ultrasound-based radiomics nomogram: A potential biomarker to predict axillary lymph node metastasis in early-stage invasive breast cancer. Eur J Radiol 2019; 119: 108658

[81] Cardoso F, Kyriakides S, Ohno S et al. Early breast cancer: ESMO Clinical Practice Guidelines for diagnosis, treatment and follow-up. Ann Oncol 2019; 30: 1194-1220

[82] Grueneisen J, Nagarajah J, Buchbender C et al. Positron Emission Tomography/Magnetic Resonance Imaging for Local Tumor Staging in Patients With Primary Breast Cancer: A Comparison With Positron Emission Tomography/Computed Tomography and Magnetic Resonance Imaging. Invest Radiol 2015; 50: 505-513

[83] Botsikas D, Kalovidouri A, Becker M et al. Clinical utility of 18F-FDG-PET/MR for preoperative breast cancer staging. Eur Radiol 2016; 26: 2297-2307

[84] Vogl WD, Pinker K, Helbich TH et al. Automatic segmentation and classification of breast lesions through identification of informative multiparametric PET/MRI features. Eur Radiol Exp 2019; 3: 18. doi:10.1186/s41747-019-0096-3

[85] Leithner D, Horvat JV, Bernard-Davila B et al. A multiparametric [18F]FDG PET/MRI diagnostic model including imaging biomarkers of the tumor and contralateral healthy breast tissue aids breast cancer diagnosis. Eur J Nucl Med Mol Imaging 2019; 46: 1878-1888

[86] Gillies RJ, Beyer T. PET and MRI: Is the Whole Greater than the Sum of Its Parts? Cancer Res 2016; 76: 6163-6166

[87] Molina-García D, García-Vicente AM, Pérez-Beteta J et al. Intratumoral heterogeneity in 18F-FDG PET/CT by textural analysis in breast cancer as a predictive and prognostic subrogate. Ann Nucl Med 2018; 32: 379388

[88] Li P, Wang X, Xu C et al. 18F-FDG PET/CT radiomic predictors of pathologic complete response ( $\mathrm{pCR}$ ) to neoadjuvant chemotherapy in breast cancer patients. Eur J Nucl Med Mol Imaging 2020; 47: 1116-1126

[89] Kirchner J, Martin O, Umutlu L et al. Impact of 18F-FDG PET/MR on therapeutic management in high risk primary breast cancer patients A prospective evaluation of staging algorithms. Eur ] Radiol 2020; 128 : 108975

[90] Ellmann S, Seyler L, Gillmann C et al. Machine Learning Algorithms for Early Detection of Bone Metastases in an Experimental Rat Model. J Vis Exp 2020. doi:10.3791/61235

[91] Gibbs P, Turnbull LW. Textural analysis of contrast-enhanced MR images of the breast. Magn Reson Med 2003; 50: 92-98

[92] Bickelhaupt S, Paech D, Kickingereder P et al. Prediction of malignancy by a radiomic signature from contrast agent-free diffusion MRI in suspicious breast lesions found on screening mammography. J Magn Reson Imaging 2017; 46: 604-616

[93] Gibbs P, Onishi N, Sadinski M et al. Characterization of Sub-1 cm Breast Lesions Using Radiomics Analysis. J Magn Reson Imaging 2019; 50: 1468-1477

[94] Milos RI, Pipan F, Kalovidouri A et al. The Kaiser score reliably excludes malignancy in benign contrast-enhancing lesions classified as BI-RADS 4 on breast MRI high-risk screening exams. Eur Radiol 2020; 30: 60526061

[95] Parekh VS, Jacobs MA. Integrated radiomic framework for breast cancer and tumor biology using advanced machine learning and multiparametric MRI. NPJ Breast Cancer 2017; 3: 43
[96] Dietzel M, Baltzer PAT. How to use the Kaiser score as a clinical decision rule for diagnosis in multiparametric breast MRI: a pictorial essay. Insights Imaging 2018; 9: 325-335

[97] Dietzel M, Baltzer PA, Vag T et al. Magnetic resonance mammography in small vs. advanced breast lesions - systematic comparison reveals significant impact of lesion size on diagnostic accuracy in 936 histologically verified breast lesions. RöFo Fortschritte Auf Dem Geb Röntgenstrahlen Nukl 2011; 183: 126-135

[98] Lambin P, Leijenaar RTH, Deist TM et al. Radiomics: the bridge between medical imaging and personalized medicine. Nat Rev Clin Oncol 2017; 14: 749-762

[99] Bluemke DA, Moy L, Bredella MA et al. Assessing Radiology Research on Artificial Intelligence: A Brief Guide for Authors, Reviewers, and Readers -From the Radiology Editorial Board. Radiology 2019; 294: 487-489

[100] Mongan J, Moy L, Kahn CE. Checklist for Artificial Intelligence in Medical Imaging (CLAIM): A Guide for Authors and Reviewers. Radiol Artif Intell 2020; 2: e200029

[101] Wu M, Zhong X, Peng Q et al. Prediction of molecular subtypes of breast cancer using BI-RADS features based on a "white box" machine learning approach in a multi-modal imaging setting. Eur J Radiol 2019; 114: $175-184$

[102] Liu C, Ding J, Spuhler K et al. Preoperative prediction of sentinel lymph node metastasis in breast cancer by radiomic signatures from dynamic contrast-enhanced MRI. J Magn Reson Imaging JMRI 2019; 49: 131140

[103] Goldhirsch A, Wood WC, Coates AS et al. Strategies for subtypes dealing with the diversity of breast cancer: highlights of the St Gallen International Expert Consensus on the Primary Therapy of Early Breast Cancer 2011. Ann Oncol 2011; 22: 1736-1747

[104] Bhargava R, Clark BZ, Carter G] et al. The healthcare value of the Magee Decision AlgorithmTM: use of Magee EquationsTM and mitosis score to safely forgo molecular testing in breast cancer. Mod Pathol 2020; 33: 1563-1570

[105] Li H, Zhu Y, Burnside ES et al. MR Imaging Radiomics Signatures for Predicting the Risk of Breast Cancer Recurrence as Given by Research Versions of MammaPrint, Oncotype DX, and PAM50 Gene Assays. Radiology 2016; 281: 382-391

[106] Boné B, Szabó BK, Perbeck LG et al. Can Contrast-Enhanced MR Imaging Predict Survival in Breast Cancer? Acta Radiol 2003; 44: 373-378

[107] Kim JH, Ko ES, Lim Y et al. Breast Cancer Heterogeneity: MR Imaging Texture Analysis and Survival Outcomes. Radiology 2016; 282: 665675

[108] Baltzer PAT, Zoubi R, Burmeister HP et al. Computer assisted analysis of MR-mammography reveals association between contrast enhancement and occurrence of distant metastasis. Technol Cancer Res Treat 2012; 11: 553-560

[109] Pinto dos Santos D, Dietzel M, Baessler B. A decade of radiomics research: are images really data or just patterns in the noise? Eur Radiol 2021; 31: 1-4

[110] Park JE, Kim D, Kim HS et al. Quality of science and reporting of radiomics in oncologic studies: room for improvement according to radiomics quality score and TRIPOD statement. Eur Radiol 2020; 30: $523-536$ 